\title{
OBSERVATIONS ON VITALLY STAINABLE RETIC- ULATION AND CHROMATIC GRANULES IN ERYTHROCYTES PRESERVED IN VITRO *
}

\author{
O. H. PERRY PEPPER, M.D. \\ PHILADELPHIA
}

These observations were made with the object of determining whether the vitally staining reticulation of young erythrocytes persists or is lost in blood preserved in vitro. In this manner it was hoped to gain some further information concerning the effect of such preservation on the erythrocyte from the point of view of the value of such cells for transfusion purposes. If it could be shown that preservation of erythrocytes in vitro for several weeks or a month did not reduce the percentage of cells in which the reticulation could be demonstrated by vital staining it might, perhaps, be assumed that the preserved erythrocytes had not aged during the period of preservation and were from this point of view as suitable for transfusion as freshly drawn cells.

Rous and Turner ${ }^{1}$ obtained the best preservation of human erythrocytes with three parts of blood in two parts of isotonic (3.8 per cent.) sodium citrate in water, and five parts of isotonic (5.4 per cent.) dextrose in water, and these observers ${ }^{2}$ found that erythrocytes thus preserved in the cold for fourteen days can be used for transfusion and will apparently remain in the circulation and function satisfactorily. Kambe and Komiya ${ }^{3}$ came to the same conclusion after studying the effect of transfusion of erythrocytes preserved in vitro up to twenty days on the appearance of polychromatophilia in the circulating blood following hemorrhage. Polychromatophilia is a regeneration phenomenon and is seen in young cells only. As a rule, it appears in twelve to twenty-four hours after hemorrhage, reaches its highest point in three or four days and disappears in a week. It was found that transfusion of preserved cells prevented the appearance of this polychromatophilia just as effectively as did transfusion of fresh cells, thus proving the vitality of the preserved cells. This did not, however, give any evidence as to the durability of the preserved cells.

\footnotetext{
* From the William Pepper Laboratory of Clinical Medicine, University of Pennsylvania.

1. Rous, P., and Turner, J. R.: The Preservation of Living Red Blood Cells in Vitro, J. Exper. M. 23:219, 1916.

2. Rous, P., and Turner, J. R.: The Preservation of Living Red Blood Cells in Vitro, J. Exper. M. 23:239, 1916.

3. Kambe, H., and Komiya. E.: Transfusion Experiment with Red Blood Corpuscles, Am. I. Physiol. 53:1 (Aıg.) 1920.
} 
There is little known about the persistence of the reticulation in erythrocytes after removal from the body. Key ${ }^{4}$ states that the reticular substance can be demonstrated in oxylated or defibrinated blood which has been shed for periods up to sixteen days if it be kept on ice. No other statements on this subject have been found in the literature.

In the present study rabbit and human bloods were employed. The rabbits were rendered anemic by bleeding or by injections of sodium oleate in order to increase the number of reticulated cells. Severe anemia was avoided for fear of altering the resistance or other characters of the erythrocytes; this point has not been sufficiently considered in experimental work. The blood was obtained under sterile precautions and either defibrinated or added to the sterile dextrose citrate solution of Rous and Turner. Samples were then placed in the ice box at $8 \mathrm{C}$. and observed at intervals. Other samples were incubated at body temperature. The count of reticulated erythrocytes was made at the time of obtaining the blood. All preparations were vitally stained with brilliant cresyl blue. For other studies of the blood Wright's stain was used.

Results.-Defibrinated rabbits' blood kept in the icebox continued to have demonstrable reticulated cells for as long as one month. After three weeks the number of reticulated cells decreased, and in some of the reticulated erythrocytes there appeared one or two small discreet granules staining blue with the cresyl blue, suggesting that the reticulation was disintegrating.

Blood taken into 3.8 per cent. sodium citrate solution almost always promptly showed a reduced number of reticulated forms as compared with the undiluted blood, but this varied considerably in various experiments. Occasionally, with human blood in citrate solution no reticulated forms could be found while with rabbit blood the reticulated erythrocytes were not lost. In one observation with rabbits' blood taken into 3.8 per cent. citrate in the proportion of three parts of blood to two parts of citrate solution and kept in the icebox reticulated erythrocytes persisted in undiminished number for a week but had disappeared three days later. Human blood usually continued to show a few reticulated forms and on one occasion these forms persisted for a month in blood which had been preserved by the Rous-Turner method. Unger ${ }^{5}$ has shown that sodium citrate even in the low percentages employed in transfusion renders the erythrocytes more fragile, but there is no evidence that this increased fragility is in any way related

4. Key, J. A.: Studies on Erythrocytes, with Special Reference to Reticulum, Polychromatophilia and Mitochondria, Arch. Int. Med. 28:511 (Nov.) 1921.

5. Unger, L. J.: Deleterious Effect of Sodium Citrate Employed in Blood Transfusion, J. A. M. A. 77:2107 (Dec. 31) 1921 . 
to the disappearance of the reticular substance. Some years ago Pepper and Peet ${ }^{6}$ were unable to demonstrate any constant difference between the reticulated and the nonreticulated forms in their resistance to hemolytic agents but no observations were made with citrate.

Incubation at body temperature of blood in citrate solution resulted in such rapid hemolysis as to make examination unprofitable after twenty-four or forty-eight hours. Defibrinated blood, however, could be studied for a number of days during incubation at body temperature. The reticulated forms rapidly disappear under these conditions; for example, in one experiment the following figures were obtained for reticulated cells: At time of bleeding, from 6 to 7 per cent.; seventeen hours after bleeding, 6 per cent.; twenty-four hours after bleeding, 4 per cent.; forty-eight hours after bleeding, 2 per cent.; seventy-two hours after bleeding, none seen.

In this same blood preserved in this icebox the percentage of reticulated forms was undiminished at the end of three weeks. In no specimen of blood left in the incubator were fully formed reticulations found after forty-eight hours but in one instance a few remnants of the reticular substance were found after sixty-six hours incubation. One would hardly be justified in drawing any conclusion from-this observation as to the probable period for which reticulation persists in young erythrocytes after they enter the circulation. It is probable, however, that the period is a short one.

In rabbits' blood preserved either in the icebox or at body temperature there appear within twenty-four or forty-eight hours in many of the cells shown to contain reticulation by vital staining with brilliant cresyl blue, one or more bright crimson spots. These number from one to, perhaps, six or eight, and vary in size from minute points up to perhaps a sixth of the diameter of the erythrocyte. In shape they are round or oval. Only when the blood has been stained with brilliant cresyl blue do they appear crimson; in the unstained wet blood they can be recognized as pale pinkish slightly refractile areas which disappear with the drying of the blood spread. With Wright's stain the spots do not take the stain but in the polychromatophilic erythrocytes there occur refractile irregular spaces which are apparently due to the same structures. In a dry preparation in which the reticular substance has been previously stained with brilliant cresyl blue these areas are seen as clear spaces often with the reticulation clumped around. With longer preservation the number of reticulated erythrocytes containing these crimson bodies increases and finally with the disappearance of the reticulated erythrocytes the crimson granules cease to be found.

6. Pepper, O. H. Perry, and Peet, Max M.: The Resistance of Reticulated Erythrocytes, Arch. Int. Med. 12:81 (July) 1913. 
These granules are in all probability the "substantia metachromatica" described by Cesaris-Demel ${ }^{7}$ in 1907 and much discussed sometimes under the term "chromatic granules of the reticular substance" in the German hematological literature since that date, especially by Schilling-Torgau ${ }^{8}$ and Pappenheim. ${ }^{9}$ Key refers to them and states that they had not been previously mentioned in American literature. $\mathrm{He}$ and others have observed them in freshly drawn, vitally stained preparations especially of anemic blood. He suggests that they may be lipoidal in nature, a view first advanced by Pappenheim but Key argues that if so the granules should be visible as refractile bodies in the unstained cell. Key did not observe such refractile bodies in the unstained cells, but in the course of the present observation this was repeatedly done. They are undoubtedly not mitochondria nor do they resemble the vacuoles which frequently occur both in reticulated and nonreticulated cells. It would seem that their increasing frequency during the preservation of erythrocytes in vitro would make it probable that they are a degenerative phenomenon of young cells. This is the view originally advanced by Cesaris-Demel. Why they should occur chiefly, if not solely, in reticulated cells has not been explained.

\section{DISCUSSION}

These results do not allow many conclusions to be drawn. There is apparently no question that sodium citrate as employed in the RousTurner method interferes to a varying degree with the demonstration by vital staining of the reticulation of young erythrocytes. But this does not prove that the erythrocyte has been injured in any manner nor that its usefulness has been impaired. Some of the cells continue to show reticulation, and these may persist for as long as a month, which is as long as the blood is considered suitable for transfusion. Defibrinated blood kept at $8 \mathrm{C}$. continues to show reticulated forms in undiminished numbers for about three weeks and this apparently argues against any aging of the corpuscles. On the other hand, the increase in the chromatic granules might be interpreted as a degenerative phenomenon and this supports the view originally. advanced by Cesaris-Demel.

7. Cesaris-Demel, A.: Studien über die roten Blutkörperchen mit den Methoden der Farbung in frischem Zustande, Folia haematol. 4: (Suppl.) 1 (Oct.) 1907.

8. Schilling-Torgau, V.: Ueber die Polychromophilie und verwandte Züstande, Folia haematol. 11:327, 1911. Arbeiten ueber die Erythrozyten, Folia haematol. 14: (Arch) 95. 1912.

9. Pappenheim, A.: Ueber Polychromophilie, Folia haematol. 9: (Zentral Organ.) 311 (June) 1910. 
It would seem that the evidence on the whole was rather in favor of the view that preservation of the blood in vitro in the cold does not of itself age the erythrocytes and so perhaps does not make them any less useful for transfusion. This would agree with the findings of others. A suspicion is raised, however, that citrate may be injurious to the erythrocytes and this effect may be the same whether it is added to prevent clotting at the moment or for the purpose of permitting preservation in vitro. This also agrees with the other evidence concerning the use of citrated blood for transfusion as presented for example by Drinker and Brittingham. ${ }^{10}$

10. Drinker, C. K., and Brittingham. H. H.: The Cause of the Reactions Following Transfusion of Citrated Blood, Arch. Int. Med. 23:133 (Feb.) 1919. 